\title{
Measurement of the photon structure function at high $Q^{2}$ at LEP
}

\section{L3 Collaboration}

M. Acciarri ${ }^{\mathrm{z}}$, P. Achard ${ }^{\mathrm{s}}$, O. Adriani ${ }^{\mathrm{p}}, \mathrm{M}$. Aguilar-Benitez ${ }^{\mathrm{y}}, \mathrm{J}$. Alcaraz $^{\mathrm{y}}$, G. Alemanni ${ }^{\mathrm{v}}$, J. Allaby ${ }^{\mathrm{q}}$, A. Aloisio ${ }^{\mathrm{ab}}$, M.G. Alviggi ${ }^{\mathrm{ab}}$, G. Ambrosi ${ }^{\mathrm{s}}$, H. Anderhub av , V.P. Andreev f,aj, T. Angelescu ${ }^{1}$, F. Anselmo ${ }^{\mathrm{i}}$, A. Arefiev ${ }^{\text {aa }}$, T. Azemoon ${ }^{\mathrm{c}}, \mathrm{T}$. Aziz ${ }^{\mathrm{j}}$, P. Bagnaia ${ }^{\mathrm{ai}}$, A. Bajo $^{\mathrm{y}}$, L. Baksay ${ }^{\mathrm{aq}}, \mathrm{A}$. Balandras ${ }^{\mathrm{d}}$, S. Banerjee ${ }^{j}$, Sw. Banerjee ${ }^{j}$, A. Barczyk ${ }^{\text {at,av }}$, R. Barillère ${ }^{q}$, L. Barone ${ }^{\text {ai }}$, P. Bartalini ${ }^{\mathrm{v}}$, M. Basile ${ }^{\mathrm{i}}$, R. Battiston ${ }^{\text {af }}$, A. Bay ${ }^{\mathrm{v}}$, F. Becattini $^{\mathrm{p}}$, U. Becker $^{\mathrm{n}}$, F. Behner ${ }^{\text {av }}$, L. Bellucci ${ }^{\mathrm{p}}$, R. Berbeco ${ }^{\mathrm{c}}$, J. Berdugo ${ }^{\mathrm{y}}$, P. Berges ${ }^{\mathrm{n}}$, B. Bertucci ${ }^{\text {af }}$, B.L. Betev ${ }^{\text {av }}$, S. Bhattacharya ${ }^{j}$, M. Biasini ${ }^{\text {af }}$, A. Biland ${ }^{\text {av }}$, J.J. Blaising ${ }^{d}$, S.C. Blyth ${ }^{\text {ag }}$, G.J. Bobbink ${ }^{\mathrm{b}}$, A. Böhm ${ }^{\mathrm{a}}$, L. Boldizsar ${ }^{\mathrm{m}}$, B. Borgia ${ }^{\mathrm{ai}}$, D. Bourilkov ${ }^{\text {av }}$, M. Bourquin ${ }^{\text {s }}$, S. Braccini ${ }^{\text {s }}$, J.G. Branson ${ }^{\text {am }}$, V. Brigljevic ${ }^{\text {av }}$, F. Brochu ${ }^{\mathrm{d}}$, A. Buffini ${ }^{\mathrm{p}}$, A. Buijs ${ }^{\text {ar }}$, J.D. Burger ${ }^{\mathrm{n}}$, W.J. Burger ${ }^{\text {af }}$, X.D. Cai ${ }^{\mathrm{n}}$, M. Campanelli ${ }^{\text {av }}$, M. Capell ${ }^{\mathrm{n}}$, G. Cara Romeo ${ }^{\mathrm{i}}$, G. Carlino ${ }^{\mathrm{ab}}$, A.M. Cartacci ${ }^{\mathrm{p}}$, J. Casaus ${ }^{\mathrm{y}}$, G. Castellini ${ }^{\mathrm{p}}$, F. Cavallari ${ }^{\text {ai }}$, N. Cavallo ${ }^{\mathrm{ak}}$, C. Cecchi ${ }^{\text {af }}$, M. Cerrada ${ }^{\mathrm{y}}$, F. Cesaroni ${ }^{\mathrm{w}}$, M. Chamizo ${ }^{\text {s }}$, Y.H. Chang ${ }^{\text {ax }}$, U.K. Chaturvedi ${ }^{r}$, M. Chemarin ${ }^{\mathrm{x}}$, A. Chen ${ }^{\text {ax }}$, G. Chen ${ }^{\mathrm{g}}$, G.M. Chen ${ }^{\mathrm{g}}$, H.F. Chen ${ }^{\mathrm{t}}$, H.S. Chen ${ }^{\mathrm{g}}$, G. Chiefari ${ }^{\mathrm{ab}}$, L. Cifarelli ${ }^{\mathrm{al}}$, F. Cindolo ${ }^{i}$, C. Civinini ${ }^{\mathrm{p}}$, I. Clare ${ }^{\mathrm{n}}$, R. Clare $^{\mathrm{n}}{ }$, G. Coignet ${ }^{\mathrm{d}}$, A.P. Colijn ${ }^{\mathrm{b}}$, N. Colino ${ }^{\mathrm{y}}$, S. Costantini ${ }^{\mathrm{e}}$, F. Cotorobai ${ }^{1}$, B. Cozzoni ${ }^{\mathrm{i}}, \mathrm{B}$. de la Cruz ${ }^{\mathrm{y}}$, A. Csilling ${ }^{\mathrm{m}}$, S. Cucciarelli ${ }^{\text {af }}$, T.S. Dai ${ }^{\mathrm{n}}$, J.A. van Dalen ${ }^{\text {ad }}$, R. D'Alessandro $^{\mathrm{p}}$, R. de Asmundis ${ }^{\text {ab }}$, P. Déglon ${ }^{\mathrm{s}}$, A. Degré ${ }^{\mathrm{d}}$, K. Deiters ${ }^{\text {at }}$, D. della Volpe ${ }^{\mathrm{ab}}$, P. Denes ${ }^{\text {ah }}$, F. DeNotaristefani ${ }^{\text {ai }}$, A. De Salvo ${ }^{\text {av }}$, M. Diemoz ${ }^{\text {ai }}$, D. van Dierendonck ${ }^{\text {b }}$, F. Di Lodovico ${ }^{\text {av }}$, C. Dionisi ${ }^{\text {ai }}$, M. Dittmar ${ }^{\text {av }}$, A. Dominguez ${ }^{\text {am }}$, A. Doria $^{\text {ab }}$, M.T. Dova ${ }^{\text {r, }}$, D. Duchesneau ${ }^{\text {d }}$, D. Dufournaud ${ }^{\text {d }}$, P. Duinker ${ }^{\text {b }}$, I. Duran ${ }^{\text {an }}$, H. El Mamouni ${ }^{\mathrm{x}}$, A. Engler ${ }^{\mathrm{ag}}$, F.J. Eppling ${ }^{\mathrm{n}}$, F.C. Erné ${ }^{\mathrm{b}}$, P. Extermann ${ }^{\mathrm{s}}$, M. Fabre ${ }^{\text {at }}$, R. Faccini ${ }^{\text {ai }}$, M.A. Falagan ${ }^{y}$, S. Falciano ${ }^{\text {q,ai }}$, A. Favara ${ }^{\mathrm{q}}$, J. Fay ${ }^{\mathrm{x}}$, O. Fedin ${ }^{\text {aj, }}$ M. Felcini ${ }^{\text {av }}$, T. Ferguson ${ }^{\text {ag }}$, F. Ferroni ${ }^{\text {ai }}$, H. Fesefeldt ${ }^{\text {a }}$, E. Fiandrini ${ }^{\text {af }}$, J.H. Field ${ }^{\text {s }}$, F. Filthaut ${ }^{\mathrm{q}}$, P.H. Fisher ${ }^{\mathrm{n}}$, I. Fisk ${ }^{\text {am }}$, G. Forconi ${ }^{\mathrm{n}}$, L. Fredj ${ }^{\mathrm{s}}$, K. Freudenreich ${ }^{\text {av }}$, C. Furetta ${ }^{z}$, Yu. Galaktionov ${ }^{\text {n,aa }}$, S.N. Ganguli ${ }^{j}$, P. Garcia-Abia ${ }^{\mathrm{e}}$, M. Gataullin ${ }^{\text {ae }}$, S.S. Gau ${ }^{\text {k }}$, S. Gentile ${ }^{\text {q,ai }}$, N. Gheordanescu ${ }^{1}$, S. Giagu ${ }^{\text {ai }}$, Z.F. Gong ${ }^{\text {}}$, G. Grenier ${ }^{\mathrm{x}}$, 
O. Grimm $^{\text {av }}$, M.W. Gruenewald ${ }^{\text {h }}$, M. Guida ${ }^{\text {al }}$, R. van Gulik ${ }^{\text {b }}$, V.K. Gupta ${ }^{\text {ah }}$, A. Gurtu ${ }^{\text {j }}$, L.J. Gutay ${ }^{\text {as }}$, D. Haas ${ }^{\text {e }}$, A. Hasan ${ }^{\text {ac }}$, D. Hatzifotiadou ${ }^{\text {i }}$, T. Hebbeker ${ }^{\text {h }}$, A. Hervé ${ }^{\mathrm{q}}$, P. Hidas ${ }^{\mathrm{m}}$, J. Hirschfelder ${ }^{\mathrm{ag}}$, H. Hofer ${ }^{\text {av }}$, G. Holzner ${ }^{\text {av }}$, H. Hoorani ${ }^{\text {ag }}$, S.R. Hou ${ }^{\text {ax }}$, Y. Hu ${ }^{\text {ad }}$, I. Iashvili ${ }^{\text {au }}$, B.N. Jin ${ }^{\mathrm{g}}$, L.W. Jones ${ }^{\mathrm{c}}$, P. de Jong ${ }^{\mathrm{b}}$, I. Josa-Mutuberría ${ }^{y}$, R.A. Khan ${ }^{\mathrm{r}}$, M. Kaur ${ }^{\mathrm{r}, 6}$, M.N. Kienzle-Focacci ${ }^{\mathrm{s}}$, D. Kim ${ }^{\text {ai }}$, J.K. Kim ${ }^{\text {ap }}$, J. Kirkby ${ }^{\text {q }}$, D. Kiss ${ }^{\text {m }}$, W. Kittel ${ }^{\text {ad }}$, A. Klimentov ${ }^{\text {naa }}$, A.C. König ${ }^{\text {ad }}$, A. Kopp ${ }^{\text {au }}$, V. Koutsenko ${ }^{\text {n,aa }}$, M. Kräber ${ }^{\text {av }}$, R.W. Kraemer ${ }^{\text {ag }}$, W. Krenz ${ }^{\text {a }}$, A. Krüger ${ }^{\text {au }}$, A. Kunin ${ }^{\text {naa }}$, P. Ladron de Guevara ${ }^{y}$, I. Laktineh ${ }^{\mathrm{x}}$, G. Landi ${ }^{\mathrm{p}}$, K. Lassila-Perini ${ }^{\text {av }}$, M. Lebeau ${ }^{q}$, A. Lebedev ${ }^{n}$, P. Lebrun ${ }^{x}$, P. Lecomte ${ }^{\text {av }}$, P. Lecoq ${ }^{q}$, P. Le Coultre ${ }^{\text {av }}$, H.J. Lee ${ }^{\text {h }}$, J.M. Le Goff ${ }^{\mathrm{q}}$, R. Leiste ${ }^{\text {au }}$, E. Leonardi ${ }^{\text {ai }}$, P. Levtchenko ${ }^{\text {aj }}$, C. Li $^{t}$, S. Likhoded ${ }^{\text {au }}$, C.H. Lin ${ }^{\text {ax }}$, W.T. Lin ${ }^{\text {ax }}$, F.L. Linde ${ }^{b}$, L. Lista $^{\text {ab }}$, Z.A. Liu ${ }^{\mathrm{g}}$, W. Lohmann ${ }^{\text {au }}$, E. Longo ${ }^{\text {ai }}$, Y.S. Lu ${ }^{\mathrm{g}}$, K. Lübelsmeyer ${ }^{\mathrm{a}}$, C. Luci ${ }^{\text {qai }}$, D. Luckey ${ }^{n}$, L. Lugnier ${ }^{\mathrm{x}}$, L. Luminari ${ }^{\text {ai }}$, W. Lustermann ${ }^{\text {av }}$, W.G. Ma ${ }^{\mathrm{t}}$, M. Maity ${ }^{\mathrm{j}}$, L. Malgeri ${ }^{\mathrm{q}}$, A. Malinin ${ }^{\mathrm{q}}$, C. Maña ${ }^{\mathrm{y}}$, D. Mangeol ${ }^{\text {ad }}$, J. Mans ${ }^{\text {ah }}$, P. Marchesini ${ }^{\text {av }}$, G. Marian ${ }^{\text {o, J.P. Martin }}{ }^{\text {x }}$, F. Marzano ${ }^{\text {ai }}$, G.G.G. Massaro ${ }^{\text {b, }}$ K. Mazumdar ${ }^{j}$, R.R. McNeil $^{\mathrm{f}}$, S. Mele ${ }^{\mathrm{q}}$, L. Merola ${ }^{\mathrm{ab}}$, M. Meschini ${ }^{\mathrm{p}}$, W.J. Metzger ${ }^{\text {ad }}$, M. von der Mey ${ }^{\text {a }}$, A. Mihul ${ }^{1}$, H. Milcent ${ }^{\mathrm{q}}$, G. Mirabelli ai, J. Mnich ${ }^{\mathrm{q}}$, G.B. Mohanty ${ }^{\mathrm{j}}$, P. Molnar ${ }^{\mathrm{h}}$, B. Monteleoni ${ }^{\mathrm{p}, 8}, \mathrm{~T}^{\mathrm{T}}$ T. Moulik ${ }^{\mathrm{j}}$, G.S. Muanza ${ }^{\mathrm{x}}$, F. Muheim ${ }^{\mathrm{s}}$, A.J.M. Muijs ${ }^{\mathrm{b}}$, M. Musy ${ }^{\mathrm{ai}}$, M. Napolitano ${ }^{\mathrm{ab}}$, F. Nessi-Tedaldi ${ }^{\text {av }}$, H. Newman ${ }^{\text {ae }}$, T. Niessen ${ }^{\text {a }}$, A. Nisati ${ }^{\text {ai }}$, H. Nowak ${ }^{\text {au }}$, G. Organtini ${ }^{\text {ai }}$, A. Oulianov ${ }^{\text {aa }}$, C. Palomares ${ }^{y}$, D. Pandoulas ${ }^{\text {a }}$, S. Paoletti ${ }^{\text {qai }}$, P. Paolucci ${ }^{\text {ab }}$, R. Paramatti ${ }^{\text {ai }}$, H.K. Park ${ }^{\text {ag }}$, I.H. Park ${ }^{\text {ap }}$, G. Pascale ${ }^{\text {ai }}$, G. Passaleva ${ }^{\text {q }}$, S. Patricelli ${ }^{\text {ab }}$, T. Paul ${ }^{\text {k }}$, M. Pauluzzi ${ }^{\text {af }}$, C. Paus ${ }^{\mathrm{q}}$, F. Pauss ${ }^{\text {av }}$, M. Pedace ${ }^{\text {ai }}$, S. Pensotti ${ }^{z}$, D. Perret-Gallix ${ }^{d}$, B. Petersen ${ }^{\text {ad }}$, D. Piccolo ${ }^{\text {ab }}$, F. Pierella $^{\mathrm{i}}$, M. Pieri $^{\mathrm{p}}$, P.A. Piroué ${ }^{\text {ah }}$, E. Pistolesi ${ }^{z}$, V. Plyaskin ${ }^{\text {aa }}$, M. Pohl ${ }^{\text {s }}$, V. Pojidaev ${ }^{\text {paa }}{ }^{\text {, H. }}$. Postema ${ }^{\text {n, }}$, J. Pothier ${ }^{\text {q }}$, N. Produit ${ }^{\text {s }}$, D.O. Prokofiev ${ }^{\text {as }}$, D. Prokofiev ${ }^{\text {aj }}$, J. Quartieri ${ }^{\text {al }}$, G. Rahal-Callot ${ }^{\mathrm{q}, \mathrm{v}}$, M.A. Rahaman ${ }^{\mathrm{j}}$, P. Raics ${ }^{\circ}$, N. Raja ${ }^{\mathrm{j}}$, R. Ramelli ${ }^{\text {av }}$, P.G. Rancoita ${ }^{\mathrm{z}}$, A. Raspereza ${ }^{\text {au }}$, G. Raven ${ }^{\text {am }}$, P. Razis ${ }^{\text {ac }}$, D. Ren ${ }^{\text {av }}$, M. Rescigno ${ }^{\text {ai }}$, S. Reucroft ${ }^{\text {k }}$, T. van Rhee ${ }^{\text {ar }}$, S. Riemann ${ }^{\text {au }}$, K. Riles ${ }^{\text {c }}$, A. Robohm ${ }^{\text {av }}$, J. Rodin ${ }^{\text {aq }}$, B.P. Roe ${ }^{\mathrm{c}}$, L. Romero ${ }^{\mathrm{y}}$, A. Rosca ${ }^{\mathrm{h}}$, S. Rosier-Lees ${ }^{\mathrm{d}}$, J.A. Rubio ${ }^{\mathrm{q}}$, D. Ruschmeier ${ }^{\text {h }}$, H. Rykaczewski ${ }^{\text {av }}$, S. Saremi ${ }^{\text {f }}$, S. Sarkar ${ }^{\text {ai }}$, J. Salicio ${ }^{\text {q }}$, E. Sanchez ${ }^{\text {q }}$, M.P. Sanders ${ }^{\text {ad }}$, M.E. Sarakinos ${ }^{\text {u }}$, C. Schäfer ${ }^{\text {q }}$, V. Schegelsky aj, S. Schmidt-Kaerst ${ }^{a}$, D. Schmitz ${ }^{\text {a }}$, H. Schopper ${ }^{\text {aw }}$, D.J. Schotanus ${ }^{\text {ad }}$, G. Schwering ${ }^{\text {a }}$, C. Sciacca ${ }^{\text {ab }}$, D. Sciarrino ${ }^{\text {s }}$, A. Seganti ${ }^{\text {i }}$, L. Servoli ${ }^{\text {af }}$, S. Shevchenko ${ }^{\text {ae, }}$, N. Shivarov ${ }^{\text {ao }}$, V. Shoutko aa, E. Shumilov ${ }^{\text {aa }}$, A. Shvorob ${ }^{\text {ae }}$, T. Siedenburg a D. Son ${ }^{\text {ap }}$, B. Smith ${ }^{\text {ag }}$, P. Spillantini ${ }^{p}$, M. Steuer ${ }^{n}$, D.P. Stickland ${ }^{\text {ah }}$, A. Stone ${ }^{\text {, }}$, B. Stoyanov ${ }^{\text {ao }}$, A. Straessner ${ }^{\text {a }}$, K. Sudhakar ${ }^{j}$, G. Sultanov ${ }^{r}$, L.Z. Sun ${ }^{\mathrm{t}}$, H. Suter ${ }^{\text {av }}$, J.D. Swain ${ }^{\mathrm{r}}$, Z. Szillasi ${ }^{\text {aq,3 }}$, T. Sztaricskai ${ }^{\text {aq,3 }}$, X.W. Tang ${ }^{\mathrm{g}}$, L. Tauscher ${ }^{\mathrm{e}}$, L. Taylor ${ }^{\mathrm{k}}$, 
B. Tellili ${ }^{\mathrm{x}}$, C. Timmermans ${ }^{\text {ad }}$, Samuel C.C. Ting ${ }^{\mathrm{n}}$, S.M. Ting ${ }^{\mathrm{n}}$, S.C. Tonwar ${ }^{\mathrm{j}}$, J. Tóth ${ }^{\mathrm{m}}$, C. Tully ${ }^{\mathrm{q}}$, K.L. Tung ${ }^{\mathrm{g}}$, Y. Uchida ${ }^{\mathrm{n}}$, J. Ulbricht ${ }^{\text {av }}$, E. Valente ${ }^{\text {ai }}$, G. Vesztergombi ${ }^{\mathrm{m}}$, I. Vetlitsky ${ }^{\text {aa }}$, D. Vicinanza ${ }^{\text {al }}$, G. Viertel ${ }^{\text {av }}$, S. Villa ${ }^{k}$, M. Vivargent ${ }^{\text {d }}$, S. Vlachos ${ }^{\mathrm{e}}$, I. Vodopianov ${ }^{\text {aj }}$, H. Vogel ${ }^{\mathrm{ag}}, \mathrm{H}$. Vogt ${ }^{\text {au }}$, I. Vorobiev ${ }^{\text {aa }}$, A.A. Vorobyov ${ }^{\text {aj }}$, A. Vorvolakos ${ }^{\text {ac }}$, M. Wadhwa ${ }^{\mathrm{e}}$, W. Wallraff ${ }^{\text {a }}$, M. Wang ${ }^{\mathrm{n}}$, X.L. Wang ${ }^{\mathrm{t}}$, Z.M. Wang ${ }^{\mathrm{t}}$, A. Weber ${ }^{\mathrm{a}}$, M. Weber ${ }^{\mathrm{a}}$, P. Wienemann ${ }^{\mathrm{a}}$, H. Wilkens ${ }^{\text {ad }}$, S.X. Wu ${ }^{\text {n}}$, S. Wynhoff ${ }^{\mathrm{q}}$, L. Xia ${ }^{\mathrm{ae}}$, Z.Z. Xu ${ }^{\mathrm{t}}$, J. Yamamoto $^{\mathrm{c}}$, B.Z. Yang ${ }^{t}$, C.G. Yang ${ }^{g}$, H.J. Yang ${ }^{g}$, M. Yang ${ }^{g}$, J.B. Ye ${ }^{t}$, S.C. Yeh ${ }^{\text {ay }}$, An. Zalite ${ }^{\text {aj }}$, Yu. Zalite ${ }^{\text {aj }}$ Z.P. Zhang ${ }^{\text {t }}$, G.Y. Zhu ${ }^{\mathrm{g}}$, R.Y. Zhu ${ }^{\text {ae }}$, A. Zichichi ${ }^{\text {i,q,r }}$, G. Zilizi ${ }^{\text {aq,3, }}$, M. Zöller ${ }^{\text {a }}$

\footnotetext{
${ }^{a}$ I. Physikalisches Institut, RWTH, D-52056 Aachen, Germany, and III. Physikalisches Institut, RWTH, D-52056 Aachen, Germany ${ }^{1}$

${ }^{\mathrm{b}}$ National Institute for High Energy Physics, NIKHEF, and University of Amsterdam, NL-1009 DB Amsterdam, The Netherlands

${ }^{\mathrm{c}}$ University of Michigan, Ann Arbor, MI 48109, USA
}

${ }^{\mathrm{d}}$ Laboratoire d'Annecy-le-Vieux de Physique des Particules, LAPP, IN2P3-CNRS, BP 110, F-74941 Annecy-le-Vieux CEDEX, France

${ }^{\mathrm{e}}$ Institute of Physics, University of Basel, CH-4056 Basel, Switzerland

${ }^{\mathrm{f}}$ Louisiana State University, Baton Rouge, LA 70803, USA

g Institute of High Energy Physics, IHEP, 100039 Beijing, China ${ }^{7}$

${ }^{\mathrm{h}}$ Humboldt University, D-10099 Berlin, Germany ${ }^{1}$

${ }^{\mathrm{i}}$ University of Bologna and INFN-Sezione di Bologna, I-40126 Bologna, Italy

${ }^{\mathrm{j}}$ Tata Institute of Fundamental Research, Bombay 400 005, India

${ }^{\mathrm{k}}$ Northeastern University, Boston, MA 02115, USA

${ }^{1}$ Institute of Atomic Physics and University of Bucharest, R-76900 Bucharest, Romania

${ }^{m}$ Central Research Institute for Physics of the Hungarian Academy of Sciences, H-1525 Budapest 114, Hungary ${ }^{2}$

${ }^{\mathrm{n}}$ Massachusetts Institute of Technology, Cambridge, MA 02139, USA

${ }^{\circ}$ KLTE-ATOMKI, H-4010 Debrecen, Hungary ${ }^{3}$

${ }^{\mathrm{p}}$ INFN Sezione di Firenze and University of Florence, I-50125 Florence, Italy

${ }^{\mathrm{q}}$ European Laboratory for Particle Physics, CERN, CH-1211 Geneva 23, Switzerland

${ }^{\mathrm{r}}$ World Laboratory, FBLJA Project, CH-1211 Geneva 23, Switzerland

${ }^{\mathrm{s}}$ University of Geneva, CH-1211 Geneva 4, Switzerland

${ }^{\mathrm{t}}$ Chinese University of Science and Technology, USTC, Hefei, Anhui 230 029, China ${ }^{7}$

u SEFT, Research Institute for High Energy Physics, P.O. Box 9, SF-00014 Helsinki, Finland

${ }^{v}$ University of Lausanne, CH-1015 Lausanne, Switzerland

${ }^{w}$ INFN-Sezione di Lecce and Universitá Degli Studi di Lecce, I-73100 Lecce, Italy

${ }^{x}$ Institut de Physique Nucléaire de Lyon, IN2P3-CNRS, Université Claude Bernard, F-69622 Villeurbanne, France

${ }^{\text {y } C e n t r o ~ d e ~ I n v e s t i g a c i o n e s ~ E n e r g e ́ t i c a s, ~ M e d i o a m b i e n t a l e s ~ y ~ T e c n o l o g i ́ c a s, ~ C I E M A T, ~ E-28040 ~ M a d r i d, ~ S p a i n ~}{ }^{4}$

${ }^{\mathrm{z}}$ INFN-Sezione di Milano, I-20133 Milan, Italy

aa Institute of Theoretical and Experimental Physics, ITEP, Moscow, Russia

${ }^{\text {ab } I N F N-S e z i o n e ~ d i ~ N a p o l i ~ a n d ~ U n i v e r s i t y ~ o f ~ N a p l e s, ~ I-80125 ~ N a p l e s, ~ I t a l y ~}$

ac Department of Natural Sciences, University of Cyprus, Nicosia, Cyprus

${ }^{\mathrm{ad}}$ University of Nijmegen and NIKHEF, NL-6525 ED Nijmegen, The Netherlands

${ }^{\text {ae }}$ California Institute of Technology, Pasadena, CA 91125, USA

af INFN-Sezione di Perugia and Universitá Degli Studi di Perugia, I-06100 Perugia, Italy

ag Carnegie Mellon University, Pittsburgh, PA 15213, USA

ah Princeton University, Princeton, NJ 08544, USA

ai INFN-Sezione di Roma and University of Rome, "La Sapienza", I-00185 Rome, Italy

${ }^{\text {aj }}$ Nuclear Physics Institute, St. Petersburg, Russia

${ }^{\mathrm{ak}}$ INFN-Sezione di Napoli and University of Potenza, I-85100 Potenza, Italy

${ }^{\text {al }}$ University and INFN, Salerno, I-84100 Salerno, Italy

am University of California, San Diego, CA 92093, USA

${ }^{\text {an }}$ Dept. de Fisica de Particulas Elementales, Univ. de Santiago, E-15706 Santiago de Compostela, Spain

ao Bulgarian Academy of Sciences, Central Lab. of Mechatronics and Instrumentation, BU-1113 Sofia, Bulgaria 


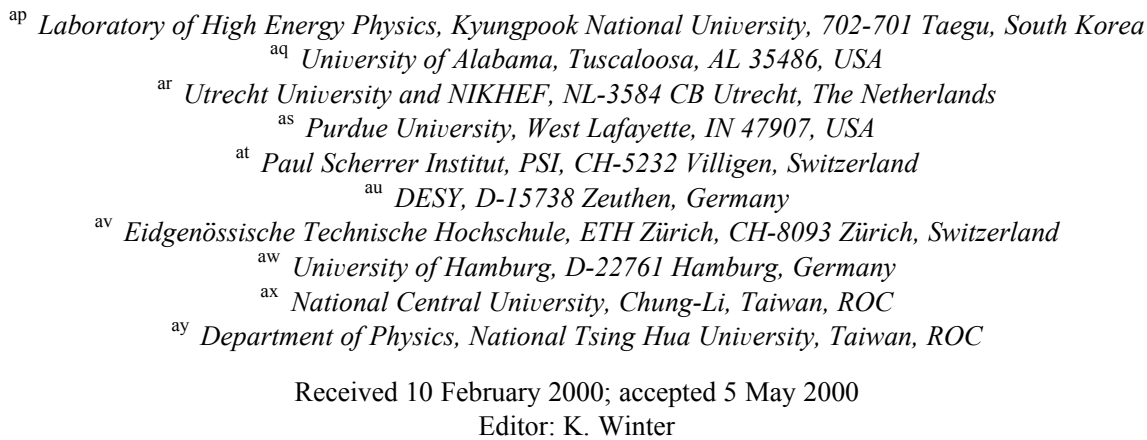

\begin{abstract}
The structure functions of real and virtual photons are derived from cross section measurements of the reaction $\mathrm{e}^{+} \mathrm{e}^{-} \rightarrow \mathrm{e}^{+} \mathrm{e}^{-}+$hadrons at LEP. The reaction is studied at $\sqrt{s} \simeq 91 \mathrm{GeV}$ with the L3 detector. One of the final state electrons is detected at a large angle relative to the beam direction, leading to $Q^{2}$ values between $40 \mathrm{GeV}^{2}$ and $500 \mathrm{GeV}^{2}$. The other final state electron is either undetected or it is detected at a four-momentum transfer squared $P^{2}$ between $1 \mathrm{GeV}^{2}$ and $8 \mathrm{GeV}^{2}$. These measurements are compared with predictions of the Quark Parton Model and other QCD based models. (C) 2000 Elsevier Science B.V. All rights reserved.
\end{abstract}

\section{Introduction}

Deep-inelastic electron scattering on a photon target is interesting because of its potential to test predictions of quantum chromodynamics (QCD) [1]. In the $Q^{2}$ domain of the present investigation, 40 $500 \mathrm{GeV}^{2}$, the photon structure function $F_{2}^{\gamma}$ is dominated by the pointlike contribution, which can be calculated by perturbative QCD, and which rises logarithmically with $Q^{2}$. In addition, there is a non-

\footnotetext{
${ }^{1}$ Supported by the German Bundesministerium für Bildung, Wissenschaft, Forschung und Technologie.

${ }^{2}$ Supported by the Hungarian OTKA fund under contract numbers T019181, F023259 and T024011.

${ }^{3}$ Also supported by the Hungarian OTKA fund under contract numbers T22238 and T026178.

${ }^{4}$ Supported also by the Comisión Interministerial de Ciencia y Tecnología.

${ }^{5}$ Also supported by CONICET and Universidad Nacional de La Plata, CC 67, 1900 La Plata, Argentina.

${ }^{6}$ Also supported by Panjab University, Chandigarh-160014, India.

${ }^{7}$ Supported by the National Natural Science Foundation of China.

${ }^{8}$ Deceased.
}

perturbative hadronic contribution, which is usually derived from a Vector Dominance (VDM) ansatz [2].

The deep-inelastic scattering of electrons on a photon target is studied by measuring the two-photon reaction $\mathrm{e}^{+} \mathrm{e}^{-} \rightarrow \mathrm{e}^{+} \mathrm{e}^{-}+$hadrons, tagged by the detection of one of the final-state electrons ${ }^{9}$ at a large four-momentum transfer $q_{1}$ (Fig. 1). The other electron usually escapes at a small scattering angle, thereby ensuring that the target photon is nearly on shell, $q_{2}^{2} \approx 0$ (single-tag events). Sometimes it is detected at small angles, with a small four-momentum transfer $q_{2}$ (double-tag events). The two virtual photons, with virtuality $Q^{2} \equiv-q_{1}^{2}$ and $P^{2} \equiv-q_{2}^{2}$, are referred to as the "probe" and "target" photons, respectively.

The differential cross section for the process $\mathrm{e}^{+} \mathrm{e}^{-} \rightarrow \mathrm{e}^{+} \mathrm{e}^{-} \gamma^{*} \gamma^{*} \rightarrow \mathrm{e}^{+} \mathrm{e}^{-} \mathrm{X}$ is given in Ref. [3]. After integration over the azimuthal angles of the outgoing electrons, it depends on four independent helicity cross sections $\sigma_{a b}$ for virtual photon-photon collisions, where $a, b=L, T$ indicate longitudinal and transverse polarizations of the probe and target pho-

\footnotetext{
${ }^{9}$ Electron stands for electron or positron throughout this paper.
} 


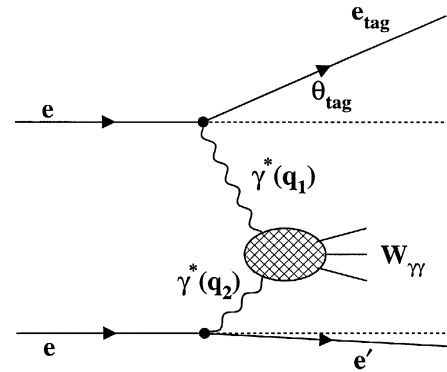

Fig. 1. Diagram of a two-photon interaction $\mathrm{e}^{+} \mathrm{e}^{-} \rightarrow \mathrm{e}^{+} \mathrm{e}^{-}+$ hadrons; $q_{1}$ and $q_{2}$ are the four-momentum vectors of the probe and target virtual photons, $W_{\gamma \gamma}=\sqrt{\left(q_{1}+q_{2}\right)^{2}}$ is the two-photon centre-of-mass energy. The electron from the lower vertex is either undetected or observed at a small angle.

tons in the $\gamma^{*} \gamma^{*}$ centre-of-mass. In the double-tag configuration investigated in this paper, $\sigma_{L L}$ is negligible in the Quark Parton Model (QPM), while in the single-tag configuration $\sigma_{T L}$ and $\sigma_{L L}$ are both negligible [3].

Introducing the hadronic mass squared $W_{\gamma \gamma}^{2}=\left(q_{1}\right.$ $\left.+q_{2}\right)^{2}$, the energy and polar angle of the first scattered electron $E_{\text {tag }}$ and $\theta_{\text {tag }}$, and the energy of the incoming electrons $E_{\text {beam }}$, the Bjorken scaling variables $x$ and $y$ are given by:

$x=\frac{Q^{2}}{Q^{2}+P^{2}+W_{\gamma \gamma}^{2}}$,

$y=1-\frac{E_{\mathrm{tag}}}{E_{\text {beam }}} \cos ^{2} \frac{\theta_{\mathrm{tag}}}{2}$.

For a real photon target $\left(P^{2}=0\right)$ the differential cross section of the deep-inelastic scattering reaction e $\gamma \rightarrow \mathrm{e}+$ hadrons can be written as

$$
\begin{aligned}
\frac{d \sigma}{d x d y}= & \frac{8 \pi \alpha^{2}}{Q^{4}} E_{\text {beam }} \\
& \times E_{\gamma}\left\{\left[1+(1-y)^{2}\right] F_{2}^{\gamma}-y^{2} F_{L}^{\gamma}\right\},
\end{aligned}
$$

where $E_{\gamma}$ is the target photon energy and

$F_{2}^{\gamma}\left(x, Q^{2}\right)=\frac{Q^{2}}{4 \pi^{2} \alpha}\left[\sigma_{T T}\left(x, Q^{2}\right)+\sigma_{\mathrm{LT}}\left(x, Q^{2}\right)\right]$

and $\quad F_{L}^{\gamma}\left(x, Q^{2}\right)=\frac{Q^{2}}{4 \pi^{2} \alpha} \sigma_{\mathrm{LT}}\left(x, Q^{2}\right)$.

By convention $F_{2}^{\gamma} / \alpha$ is measured, where $\alpha$ is the fine-structure constant. In order to obtain the $\mathrm{e}^{+} \mathrm{e}^{-}$ cross section a convolution with the flux function of the target photon is necessary. For the single-tag condition the $F_{L}^{\gamma}$ dependent term in Eq. (2) is less than $5 \%$ in the $\mathrm{QPM}^{10}$. In the double-tag measurement $\sigma_{T L}$ cannot be neglected; consequently one can only measure an effective structure function

$F_{\mathrm{eff}}^{\gamma}=\frac{Q^{2}}{4 \pi^{2} \alpha}\left(\sigma_{T T}+\sigma_{\mathrm{LT}}+\sigma_{T L}+\sigma_{L L}\right)$.

The photon structure function has been measured at various $Q^{2}$ values at the PETRA, PEP and TRISTAN accelerators [4] and at LEP [5-8]. The structure function for double tag was first measured by the PLUTO collaboration [9]. Here we report on the analysis of events with $Q^{2}$ in the range $40-500 \mathrm{GeV}^{2}$ and $P^{2}$ either close to zero or in the range $1-8 \mathrm{GeV}^{2}$. The data were collected by the L3 detector during the years $1991-1995$ at $\sqrt{s}=89-92 \mathrm{GeV}$ with a total integrated luminosity of $120 \mathrm{pb}^{-1}$.

\section{Kinematic fitting}

A new feature in the present work is a fit to two-photon kinematics imposed on each event. Inputs to the fit are the measurements of the kinematic variables of the hadrons and of the scattered electrons, constrained to four-momentum conservation. The single- and double-tag cases are treated differently.

In the single-tag case, the event plane is taken to be the scattering plane, defined by the directions of the tagged electron and the beam. In the double-tag case, the event plane is defined by the direction of the vector sum of the momenta of the tagged electrons and by the beam direction. The components of the momentum vectors in the event plane are labelled $p_{z}$ and $p_{\text {in }}$, parallel and perpendicular to the beam respectively; $p_{\text {out }}$ is the component perpendicular to this plane. The positive $z$ direction is defined in the direction of the tagged electron. From the energy and momenta of the hadronic system, $\left(E^{h}, p^{h}\right)$, of the scattered positron, $\left(E^{+}, p^{+}\right)$, and of

\footnotetext{
${ }^{10}$ For $y<0.7$ the factor $y^{2} /\left(1+(1-y)^{2}\right)$ is on average of the order of 0.2 and the QPM predicts $F_{L}^{\gamma} / F_{2}^{\gamma}$ to be smaller than 0.25 . This leads to $\left[y^{2} /\left(1+(1-y)^{2}\right)\right] F_{L}^{\gamma} / F_{2}^{\gamma}<0.05$.
} 
the scattered electron, $\left(E^{-}, p^{-}\right)$, three energy and momentum constraints are defined:

$$
\begin{aligned}
& C_{1}=E^{-}+E^{+}+E^{h}-2 E_{\text {beam }}+2 K_{\text {rad }}, \\
& C_{2}=p_{z}^{-}+p_{z}^{+}+p_{z}^{h}, \quad C_{3}=p_{\text {in }}^{-}+p_{\text {in }}^{+}+p_{\text {in }}^{h} .
\end{aligned}
$$

The term $K_{\text {rad }}$ describes the average energy carried away by unobserved initial state radiative photons (ISR). In the fit, the following expression is minimized with respect to three components of the fourmomentum vector of the hadronic system:

$\chi^{2}=\sum_{i, j=1}^{3} V_{i, j}^{-1} \Delta p_{i} \Delta p_{j}+\sum_{k=1}^{3} W_{k} C_{k}^{2}$.

Four energy and momentum differences are defined as:

$$
\begin{aligned}
& \Delta p_{1}=E^{h}-\sum_{l} E_{l}^{h}, \quad \Delta p_{2}=p_{z}^{h}-\sum_{l} p_{z, l}^{h}, \\
& \Delta p_{3}=p_{\text {in }}^{h}-\sum_{l} p_{\text {in }, l}^{h}, \quad \Delta p_{4}=p_{\text {out }}^{h}-\sum_{l} p_{\text {out }, l}^{h},
\end{aligned}
$$

where the index $l$ runs over all detected hadrons and $E^{h}, p_{z}^{h}, p_{\text {in }}^{h}$ are the fit quantities. The $3 \times 3$ error matrix, $V$, of the hadronic energy measurement is calculated from the individual cluster energy and momentum measurements by taking into account errors in energy measurements and uncertainties in the hadron directions. The constraints $C_{k}$ are applied with constant weight factors $W_{k}$. These weight factors reflect the accuracy of the measurements on the energies of the tagged electrons and the spread introduced by radiative corrections. The distribution of $\Delta p_{4}$ is not used in the fit but to compare the hadronic energy resolution between data and Monte Carlo.

In the single-tag case the unseen electron is assumed to have zero scattering angle. As one of the electron momenta is unknown, the constraints $C_{1}$ and $C_{2}$ are combined into:

$$
C_{12}=\frac{E^{h}+p_{z}^{h}}{2}-E_{\text {beam }}+K_{\mathrm{rad}}+\frac{E_{\mathrm{tag}}+p_{z, \mathrm{tag}}}{2} .
$$

Most two-photon Monte Carlo programs have no provision for ISR. The value of $K_{\text {rad }}$, estimated by a program of Berends, Daverveldt and Kleiss [10], is of the order of $1.0-1.5 \mathrm{GeV}$ for a beam energy of $45.6 \mathrm{GeV}$. It increases logarithmically with the tagging angle. In fits to the data such losses are taken into account by using $K_{\mathrm{rad}}=1.5 \mathrm{GeV}$ for the large- angle electron and $K_{\mathrm{rad}}=1.0 \mathrm{GeV}$ for the small-angle electron in double-tag events.

Before the fit the mean values of $\Delta p_{i}$, with $i=1-3$, are adjusted to zero by scaling the hadronic energies and momenta. The scale factor accounts for the average reduction in hadron energy from the loss of particles. The hadron energy resolution is estimated from the widths of the constraint distributions. The scaling procedure improves the measurement of the visible hadronic mass.

The kinematic fit, applied to each event, determines the fitted value of the hadronic invariant mass and, for single-tag events, the energy of the unobserved electron, $E_{\mathrm{mis}}$ :

$W_{\gamma \gamma, \text { fit }}^{2}=E^{h 2}-p_{\text {in }}^{h 2}-p_{z}^{h 2}$,

$E_{\text {mis }}=E_{\text {beam }}-\frac{0.5\left(W_{\gamma \gamma, \text { fit }}^{2}+Q^{2}\right)}{2 E_{\text {beam }}-\left(E_{\text {tag }}+p_{z, \text { tag }}\right)}$.

The correlation between the generated values of $W_{\gamma \gamma}$ and $x$ and their fitted values for single-tag $\mathrm{q} \overline{\mathrm{q}}$
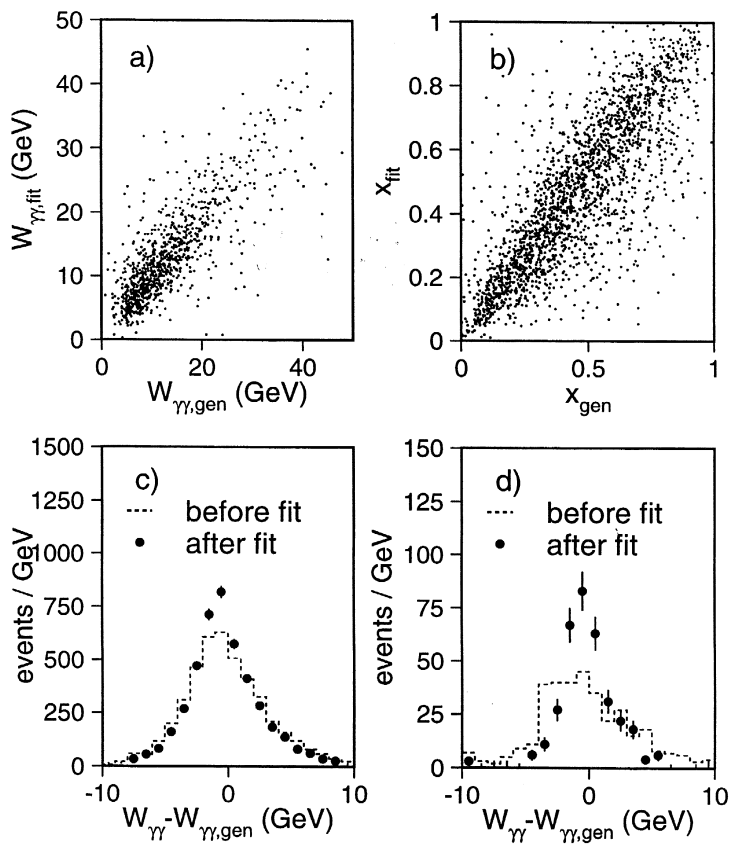

Fig. 2. (a) Correlation between the generated value of $W_{\gamma \gamma}$ and the measured value after the kinematic fit. In all cases the JAMVG Monte Carlo has been used. (b) Correlation between the generated value of $x$ and the measured value after the kinematic fit. (c) The hadronic mass resolution before and after the fit for single-tag events. (d) The hadronic mass resolution before and after the fit for double-tag events. 
events is displayed in Fig. 2; their relation is approximately linear. The kinematical fit improves the hadronic mass resolution by about $12 \%$ in single-tag events and by $30 \%$ in double-tag events. After the kinematical fit, the one sigma mass resolution is about $3.5 \mathrm{GeV}$ for single-tag and $2.7 \mathrm{GeV}$ for doubletag events; the resolution in $x$ is 0.13 for single-tag and 0.10 for double-tag events. The resolution is constant in $x$ and $Q^{2}$ in the ranges covered by the experiment.

\section{Event selection}

A detailed description of the L3 detector and its performance is given in Ref. [11]. The analysis in this paper is based on the central tracking system, the high resolution electromagnetic calorimeters and the hadron calorimeters. The electron scattered at high $Q^{2}$ is observed in the endcap electromagnetic calorimeter at a polar angle $\theta$ between 200 and 700 mrad with respect to the direction of one of the beams. The other electron remains either undetected or it is observed in one of the small-angle electromagnetic calorimeters in a fiducial region $29 \mathrm{mrad}$ $\leq \theta \leq 67 \mathrm{mrad}$. The hadronic energy and momentum and the visible mass are derived from the energy clusters in the electromagnetic and hadron calorimeters. Energy clusters in the small-angle calorimeters are also included if their energy is less than $18 \mathrm{GeV}$.

The single-tag events are accepted by two independent triggers, a charged particle track trigger [12] and a calorimetric energy trigger [13]. The average efficiency of the combination of both triggers, deduced from the data, is $95 \pm 1 \%$. It decreases to $85 \%$ at the lowest accepted visible hadronic mass of $3 \mathrm{GeV}$. For the double-tag events the energy trigger also accepts a small-angle electron in coincidence with one charged particle. This yields a trigger efficiency larger than $98 \%$ for double-tag events.

The selection of the process $\mathrm{e}^{+} \mathrm{e}^{-} \rightarrow \mathrm{e}^{+} \mathrm{e}^{-}+$ hadrons has been guided by fully simulated event samples from several $\gamma \gamma$ Monte Carlo generators. The JAMVG [14] $\gamma \gamma$ generator is based on an exact calculation of the multiperipheral diagrams. The $\gamma \gamma$ $\rightarrow \mathrm{u} \overline{\mathrm{u}}$ and $\gamma \gamma \rightarrow \mathrm{c} \overline{\mathrm{c}}$ channels are generated separately with $m_{\mathrm{u}}=0.325 \mathrm{GeV}$ and $m_{\mathrm{c}}=1.6 \mathrm{GeV}$. The contributions from d- and s-quarks are taken into ac- count by a multiplicative factor $9 / 8$ to the uu cross section. PHOJET [15] is an event generator, within the Dual Parton Model framework. The photon is considered as a superposition of a "bare photon" and virtual hadronic states. To separate soft from hard processes, a transverse momentum cutoff at $2.5 \mathrm{GeV}$ is applied to all the partons of the pointlike interactions [16]. TWOGAM [17] generates three different $\gamma \gamma$ processes separately: the QPM, soft hadronic VDM and the QCD resolved photon contribution with a transverse momentum cutoff at $2.5 \mathrm{GeV}$.

The dominant background processes are $\mathrm{e}^{+} \mathrm{e}^{-} \rightarrow$ hadrons, simulated by JETSET [18], $\mathrm{e}^{+} \mathrm{e}^{-} \rightarrow \tau^{+} \tau^{-}$ simulated by KORALZ [19] and $\mathrm{e}^{+} \mathrm{e}^{-} \rightarrow \mathrm{e}^{+} \mathrm{e}^{-} \tau^{+} \tau^{-}$, simulated by JAMVG.

Single-tag hadronic events are selected as follows:

1. The scattered electron candidate is an electromagnetic cluster with an energy greater than $30 \%$ of the beam energy.

2. At least three tracks are seen in the central tracking system.

3. The visible hadronic mass $W_{\gamma \gamma \text {,vis }}$ is greater than $3 \mathrm{GeV}$.

4. The hadronic transverse momentum component, $p_{\text {out }}^{h}$, is less than $5 \mathrm{GeV}$ and $p_{\text {in }}^{h} \cdot p_{\text {in }}^{\text {tag }}<0$, where $p^{\text {tag }}$ stands for the momentum of the tagged electron.

5. The event rapidity, $\eta$, must be greater than 0.4 , see Fig. $3 \mathrm{a} ; \eta$ is defined by

$\eta=\frac{1}{2} \ln \frac{\sum_{i}\left(E_{i}+p_{z, i}\right)}{\sum_{i}\left(E_{i}-p_{z, i}\right)}$,

where $i$ runs over the calorimetric clusters, including that of the scattered electron. This requirement suppresses the annihilation reaction, where the total longitudinal momentum of the event is close to zero. In the two-photon reaction it can be of the order of $40 \mathrm{GeV}$ because of the momentum of the unobserved electron.

6. The $\chi^{2}$ probability in the kinematic fit is greater than $10^{-5}$.

7. The momentum of the unobserved electron must be greater than $26 \mathrm{GeV}$. 

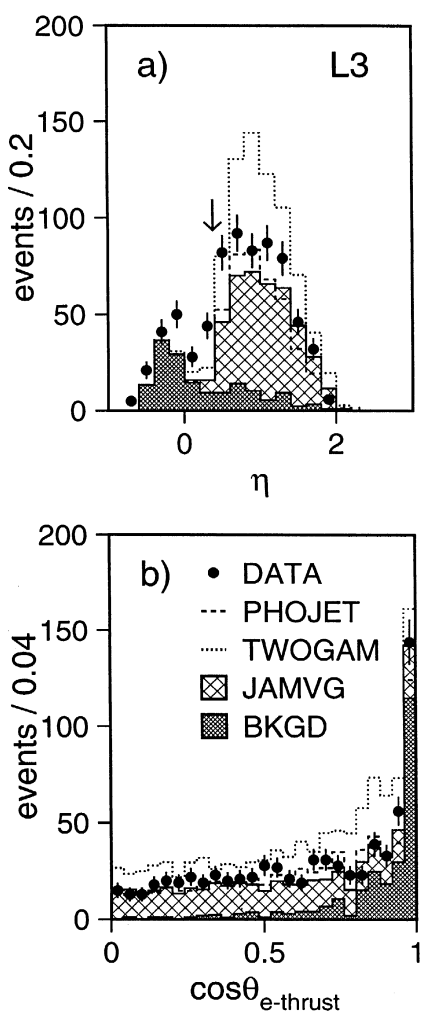

Fig. 3. (a) The distribution of the event rapidity, $\eta$, for single-tag events; the arrow indicates the cut at $\eta=0.4$. (b) The cosine of the angle between the tagging electron and the thrust direction in the $\gamma \gamma$ centre-of-mass for single-tag events. The cut on $\cos \theta_{\mathrm{e}-\text { thrust }}$ is mass dependent, see the text. All selection cuts are fulfilled, except the one on the plotted variable. The Monte Carlo distributions, JAMVG, PHOJET, TWOGAM and the background from annihilation and two-photon $\tau$-pair production, are normalized to the integrated luminosity of the data.

8. A thrust $T$ and a thrust axis $\hat{n}$ are defined by maximising the linear sum of projected hadronic cluster momenta along $\hat{n}[20]$

$$
T=\frac{\operatorname{Max} \sum_{i}\left|\boldsymbol{p}_{i}^{h, *} \cdot \hat{n}\right|}{\sum_{i}\left|\boldsymbol{p}_{i}^{h, *}\right|} .
$$

Here $\boldsymbol{p}_{i}^{h, *}$ refers to the hadronic cluster momentum in the $\gamma \gamma$ centre-of-mass. A variable cut is applied on the maximum allowed value of the cosine of the electron-thrust angle in the $\gamma \gamma$ centre-of-mass if the hadronic mass is greater than $10 \mathrm{GeV}$; its value is $0.92,0.80,0.70,0.60$ for masses smaller than $20,30,40,50 \mathrm{GeV}$, respectively. This cut discriminates against the annihilation reaction, in particular it removes $\mathrm{e}^{+} \mathrm{e}^{-} \rightarrow \mathrm{b} \bar{b}$ events, where the b-quark decays semileptonically. In that case the electrons are emitted predominantly in the thrust direction, whereas no such correlation is present in the two-photon reaction (see Fig. 3b).

Double-tag hadronic events are selected in a similar way to single-tag events. The small-angle scattered electron candidate must have an energy greater than $40 \%$ of the beam energy, and no cut is applied to the event rapidity.

Table 1 shows the numbers of events selected in the range $40 \mathrm{GeV}^{2} \leq Q^{2} \leq 500 \mathrm{GeV}^{2}$ and $3 \mathrm{GeV} \leq$ $W_{\gamma \gamma \text {,fit }} \leq 50 \mathrm{GeV}$. The largest contamination in the single-tag sample comes from two-photon $\tau$-pair production. The influence of the annihilation background in single-tag events is checked by comparing the data taken at the $\mathrm{Z}$ peak with the off-peak data ( $25 \%$ of the sample) where it is about a factor three smaller than on the peak. Within the limited accu-

Table 1

Numbers of selected events and Monte Carlo predictions normalized to the luminosity of the data.

\begin{tabular}{|c|c|c|}
\hline Data & $\begin{array}{l}\text { Single tag events } \\
496\end{array}$ & $\begin{array}{l}\text { Double tag events } \\
43\end{array}$ \\
\hline \multicolumn{3}{|l|}{ Background } \\
\hline $\mathrm{e}^{+} \mathrm{e}^{-} \rightarrow \mathrm{e}^{+} \mathrm{e}^{-} \tau^{+} \tau^{-}$ & $24 \pm 6$ & $<1$ \\
\hline $\mathrm{e}^{+} \mathrm{e}^{-} \rightarrow \mathrm{q} \overline{\mathrm{q}}$ & $12 \pm 7$ & $<2$ \\
\hline $\mathrm{e}^{+} \mathrm{e}^{-} \rightarrow \tau^{+} \tau^{-}$ & $4 \pm 2$ & $<1$ \\
\hline Data - Background & $456 \pm 24$ & $43 \pm 7$ \\
\hline \multicolumn{3}{|l|}{ JAMVG } \\
\hline $\mathrm{uu}, \mathrm{d} \overline{\mathrm{d}}, \mathrm{s} \overline{\mathrm{s}}$ & $233 \pm 2$ & $15 \pm 1$ \\
\hline $\mathrm{c} \overline{\mathrm{c}}$ & $111 \pm 1$ & $10 \pm 0$ \\
\hline Total & $344 \pm 2$ & $25 \pm 1$ \\
\hline PHOJET & $346 \pm 2$ & $48 \pm 1$ \\
\hline \multicolumn{3}{|l|}{ TWOGAM } \\
\hline QPM & $335 \pm 5$ & $24 \pm 1$ \\
\hline VDM & $126 \pm 3$ & $9 \pm 1$ \\
\hline QCD & $162 \pm 1$ & $29 \pm 1$ \\
\hline Total & $624 \pm 6$ & $62 \pm 2$ \\
\hline
\end{tabular}


racy the off-peak sample is consistent with the total selected sample.

\section{Comparison of data with models}

The data are compared to Monte Carlo expectations in Table 1 and in Figs. 4, 5 and 6. In Fig. 4 kinematic properties in the $\gamma \gamma$ centre-of-mass of the selected single-tag events are compared in shape to Monte Carlo predictions. For this purpose the Monte Carlo distributions are normalized to the same number of events as the data. In 4a the thrust exhibits a wide distribution, which is adequately described by JAMVG (QPM) and less well by the PHOJET and TWOGAM models. In Figs. $4 \mathrm{~b}$ and $4 \mathrm{c}$ (for $x<0.2$ ) the distribution of the cosine of the polar angle of the thrust direction with respect to the $\gamma \gamma$ axis $\left(\theta_{\text {thrust }}\right)$ shows forward peaking in good agreement with the JAMVG simulation. A diffractive forward peak, pre-
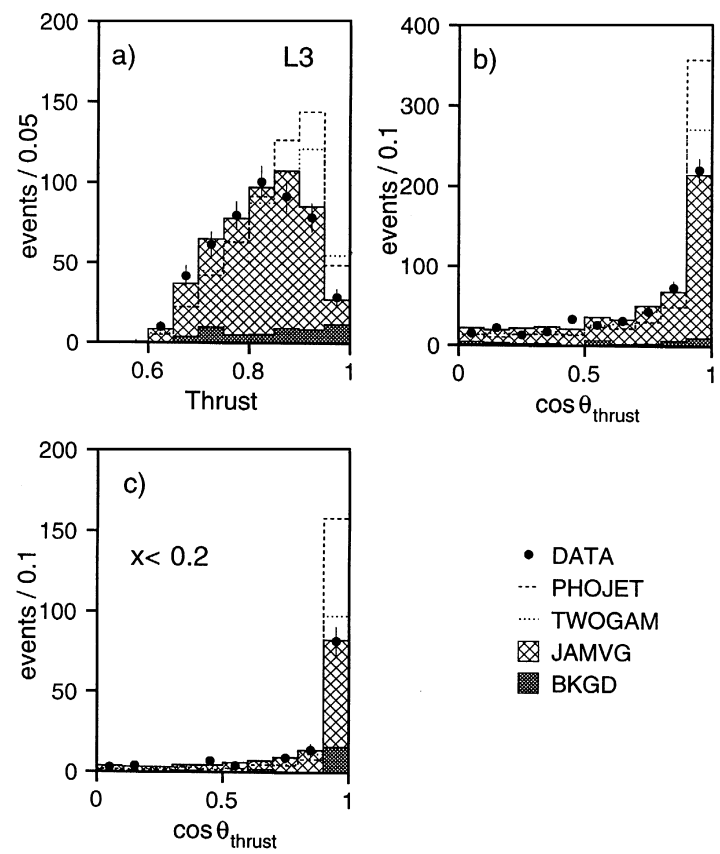

Fig. 4. The distributions of (a) thrust, (b) $\cos \theta_{\text {thrust }}$ and (c) $\cos \theta_{\text {thrust }}$ for events with $x_{\text {fit }}<0.2$ in the $\gamma \gamma$ centre-of-mass frame for single-tag. The JAMVG, PHOJET and TWOGAM contributions are summed with the background and scaled to have the same number of simulated events as in the data.
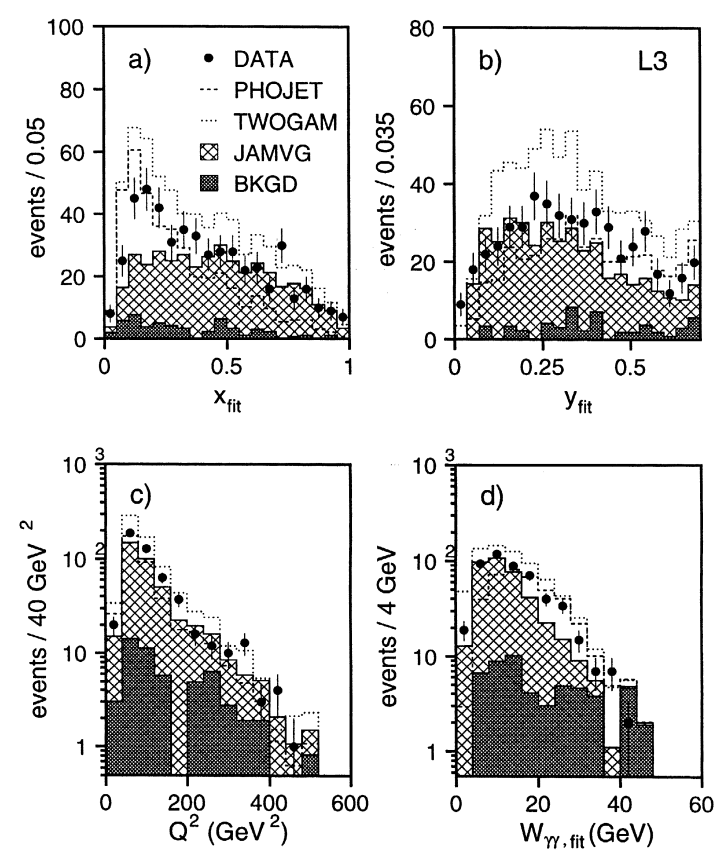

Fig. 5. The (a) $x_{\text {fit }}$, (b) $y_{\text {fit }}$, (c) $Q^{2}$, (d) $W_{\gamma \gamma \text {,fit }}$, distributions for single-tag events. The data are compared to the JAMVG, PHOJET and TWOGAM models. The predictions include the estimated background distributions. The Monte Carlo distributions are normalized to the integrated luminosity of the data.

dicted by PHOJET and TWOGAM, is not observed in the data.

It should be noted that the compatibility of the QPM angular distributions with the data does not exclude the contribution from QCD processes, such as photon-gluon fusion and $\gamma \mathrm{q} \rightarrow \mathrm{gq}$ which have similar angular distributions [21], but indicates a low contribution of VDM and diffractive processes.

Figs. 5a-d show the distributions of $x_{\text {fit }}, y_{\text {fit }}, Q^{2}$ and $W_{\gamma \gamma \text {,fit }}$, together with Monte Carlo background estimations, for the selected single-tag events, in comparison with Monte Carlo predictions normalised to the data luminosity. Although the number of events expected by JAMVG is too small, the $Q^{2}$ distribution follows the predicted shape. The hadronic mass $W_{\gamma \gamma \text {,fit }}$ presents an excess of data over JAMVG predictions at masses above $20 \mathrm{GeV}$. The $x_{\text {fit }}$ distribution is limited by the maximum mass observed, $W_{\gamma \gamma, \mathrm{fit}} \approx 50 \mathrm{GeV}$, and by the minimum mass of $W_{\gamma \gamma, \mathrm{fit}}=3 \mathrm{GeV}$ and by the restricted $Q^{2}$ range from $40 \mathrm{GeV}^{2}$ to $500 \mathrm{GeV}^{2}$. The data agree with the 

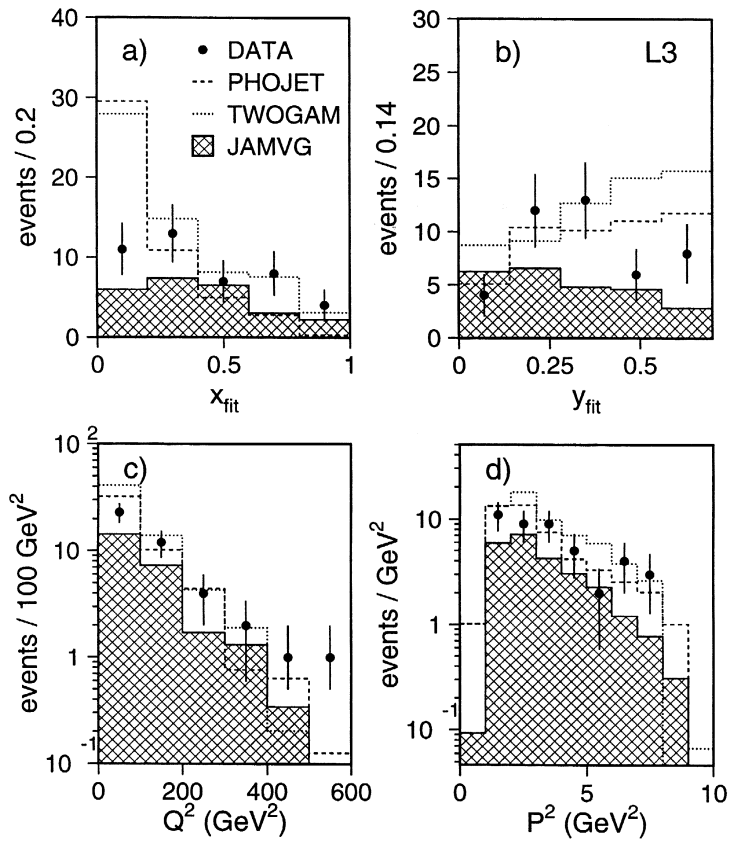

Fig. 6. The (a) $x_{\text {fit }}$, (b) $y_{\text {fit }}$, (c) $Q^{2}$, (d) $P^{2}$ distributions for double-tag events. The data are compared to the JAMVG, PHOJET and TWOGAM predictions. Backgrounds are estimated to be negligible. The Monte Carlo distributions are normalized to the integrated luminosity of the data.

prediction of JAMVG for $x_{\text {fit }}>0.5$. For lower $x_{\text {fit }}$ values the QPM alone is insufficient to reproduce the data. The excess of events over the JAMVG prediction at $x_{\text {fit }}<0.2$ and $y_{\text {fit }}>0.3$ is compatible with the existence of a QCD contribution in the measured $Q^{2}$ range. TWOGAM predicts too many events at all $x_{\text {fit }}$ values. PHOJET agrees with the data at low $x_{\text {fit }}$, but not at high $x_{\text {fit }}$ because this program suppresses also the QPM diagram with the cutoff on the quark transverse momentum $\left(p_{t}>2.5 \mathrm{GeV}\right)$. Also the $\mathrm{c} \overline{\mathrm{c}}$ production is suppressed in PHOJET, whereas the JAMVG program predicts a substantial charm contribution in both single- and double-tag events.

The double-tag events have a negligible background from Z-decay and $\tau$-pairs. Figs. $6 \mathrm{a}-\mathrm{d}$ show the distributions for $x_{\text {fit }}, y_{\text {fit }}, Q^{2}$ and $P^{2}$, together with the Monte Carlo expectations. Also here the QPM model implemented in JAMVG predicts too few events, but the kinematic distributions, such as the thrust, the thrust angular distributions and the $p_{t}$ distribution of the energy clusters, are well reproduced. PHOJET and TWOGAM expectations exceed the data at low $x_{\text {fit }}$ values, thus indicating that the QCD contributions are overestimated.

\section{Photon structure functions}

\subsection{Single tag}

The JAMVG generator reproduces the shape of the kinematic distributions. Therefore it provides the basis for calculating the acceptance and it can be used to unfold the $x_{\text {fit }}$ distributions to distributions in true values of $x$. After subtraction of background, the single-tag data are divided in five $x_{\text {fit }}$ bins, and unfolded into $x$ bins with the SVD method [22]. The unfolding procedure is considered to be satisfactory if the $x_{\text {fit }}$ distribution, calculated from the unfolded result, reproduces the measured $x_{\text {fit }}$ distribution within its statistical errors. The acceptance has a maximum of about $60 \%$ at $x=0.4$ and minima of about $40 \%$ at both ends of the $x$ interval.

The value of $F_{2}^{\gamma} / \alpha$ is obtained by comparing the experimental $x$ distribution to the generated one. The ratios, given in Table 2, are applied to the QPM analytical expression for $F_{2}^{\gamma}(x) / \alpha$, obtained from the $\sigma_{T T}$ and $\sigma_{\mathrm{LT}}$ formulæ, given in Appendix $\mathrm{E}$ of Ref. [3], calculated for every $\langle x\rangle$ value at the average $Q^{2}$ of the data distribution, $Q^{2}=120 \mathrm{GeV}^{2}$, and at $P^{2}=0$. The QPM estimates that the structure function, calculated at the the mean value of the target photon virtuality $P^{2}=0.014 \mathrm{GeV}^{2}$, is only 0.2 $\%$ smaller than the value expected for a real photon, this effect is therefore neglected. The result is shown in Fig. 7 and in Table 2. The correlation between the statistical errors, introduced by the unfolding procedure, is small. The correlation matrix is also given in Table 2.

Estimates of systematic uncertainties from various sources on each data point are summarized in Table 3 , averaged over the entire $x$ and $Q^{2}$ range. The misidentification of hadrons as tagged electrons is small due to the requirement of a substantial tag energy; the corresponding uncertainty on the tag selection is estimated by comparing the results of the different generators. The model dependence in the acceptance calculation and in the selection efficiency 
Table 2

$F_{2}^{\gamma} / \alpha$ as a function of $x$ for real photons at $Q^{2}=120 \mathrm{GeV}^{2}$, from single-tag events.

\begin{tabular}{lrrrrr}
\hline$x$ range & $0.05-0.2$ & $0.2-0.4$ & $0.4-0.6$ & $0.6-0.8$ & $0.8-0.98$ \\
$\langle x\rangle$ & 0.13 & 0.30 & 0.50 & 0.70 & 0.89 \\
\hline Ratio to QPM & $2.26 \pm 0.27$ & $1.56 \pm 0.16$ & $1.03 \pm 0.16$ & $0.95 \pm 0.15$ & $0.18 \pm 0.25$ \\
& & & & \\
$F_{2}^{\gamma}(x) / \alpha$ & $0.66 \pm 0.08$ & $0.81 \pm 0.08$ & $0.76 \pm 0.12$ & $0.85 \pm 0.14$ & $0.91 \pm 0.19$ \\
Systematic uncertainty & 0.06 & 0.08 & 0.07 & -0.02 & 0.09 \\
Correlation & 1 & 0.18 & -0.19 & -0.18 & 0.09 \\
matrix & & 1 & 0.03 & 0.09 & -0.21 \\
& & & & 0.04 & 1
\end{tabular}

as function of $x_{\text {fit }}$ is also evaluated from the Monte Carlo generators. The average spread of $4.6 \%$ is
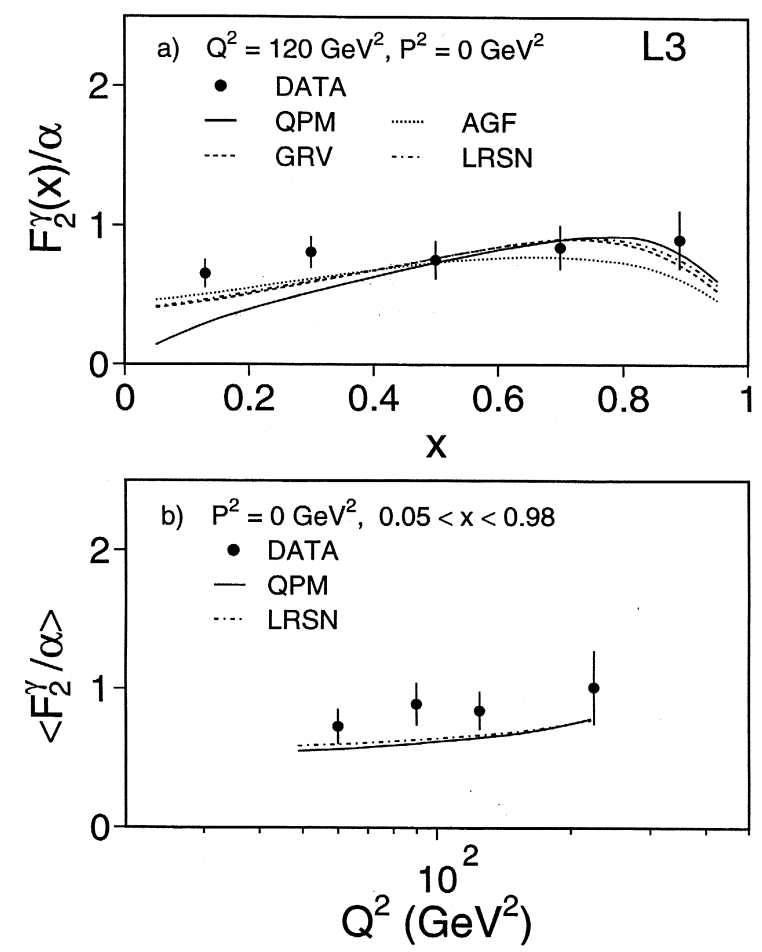

Fig. 7. (a) The structure function $F_{2}^{\gamma} / \alpha$ for real photons at $Q^{2}=120 \mathrm{GeV}^{2}$ compared with the QPM calculation and the QCD calculations GRV, AGF and LRSN described in the text. (b) Dependence on $Q^{2}$ of $F_{2}^{\gamma} / \alpha$ averaged over $x=0.05-0.98$ for single-tag data, compared with the QPM indicated by a full line and with the LRSN calculation described in the text. The errors are statistical and systematic added in quadrature. included in the systematic uncertainty. JAMVG includes only the multiperipheral two-photon diagram. The contributions from the bremsstrahlung, annihilation and conversion diagrams missing in JAMVG have been estimated for the single-tag configuration with the program DIAG36 [23]. They are small, and consistent with zero with a $2.4 \%$ uncertainty. The systematic uncertainties in the event selection as defined in Section 3 have been estimated by varying the cut parameters. An estimate of the effect of radiative corrections on the cross section has been made by Laenen and Schuler [24]. In the region of interest they find a reduction of the cross section of the order of $2.6-3.8 \%$. The average is taken to be the systematic uncertainty. No radiative correction is applied to the data. The annihilation background has been estimated by varying the cut on the variable $\eta$,

Table 3

Systematic relative uncertainties on $F_{2}^{\gamma}\left(x, Q^{2}\right) / \alpha$ and $F_{\text {eff }}^{\gamma}\left(x, Q^{2}, P^{2}\right) / \alpha$.

\begin{tabular}{lll}
\hline $\begin{array}{l}\text { Source of systematic } \\
\text { uncertainty }\end{array}$ & $\begin{array}{l}\text { Single } \\
\text { tag }\end{array}$ & $\begin{array}{l}\text { Double } \\
\text { tag }\end{array}$ \\
\hline Monte Carlo statistics & $1.7 \%$ & $5.8 \%$ \\
Tag selection & $1.0 \%$ & $1.4 \%$ \\
Hadron acceptance & $4.6 \%$ & $4.6 \%$ \\
Triggering & $1.0 \%$ & $1.0 \%$ \\
Event selection & $3.0 \%$ & $1.6 \%$ \\
Radiative corrections & $3.2 \%$ & $3.2 \%$ \\
Modelling of background & $4.4 \%$ & $4.7 \%$ \\
Unfolding & $5.3 \%$ & $8.1 \%$ \\
Total & & \\
\hline
\end{tabular}


Table 4

$Q^{2}$ dependence of $F_{2}^{\gamma} / \alpha$ for real photons for various $x$ intervals. In the last row the structure function value is given for the total sample of single-tag events.

\begin{tabular}{llll}
\hline$\left\langle Q^{2}\right\rangle$ & $\left\langle F_{2}^{\gamma} / \alpha\right\rangle$ & $\left\langle F_{2}^{\gamma} / \alpha\right\rangle$ & $\left\langle F_{2}^{\gamma} / \alpha\right\rangle$ \\
$\left(\mathrm{GeV}^{2}\right)$ & $x=0.05-0.98$ & $x=0.3-0.8$ & $x=0.1-0.6$ \\
\hline 60 & $0.73 \pm 0.11 \pm 0.07$ & $0.66 \pm 0.09 \pm 0.06$ & $0.63 \pm 0.06 \pm 0.06$ \\
90 & $0.89 \pm 0.13 \pm 0.09$ & $0.79 \pm 0.14 \pm 0.08$ & $0.92 \pm 0.14 \pm 0.09$ \\
125 & $0.85 \pm 0.11 \pm 0.09$ & $0.88 \pm 0.12 \pm 0.08$ & $0.86 \pm 0.14 \pm 0.08$ \\
225 & $1.01 \pm 0.25 \pm 0.10$ & $1.18 \pm 0.22 \pm 0.11$ & $0.91 \pm 0.30 \pm 0.09$ \\
120 & $0.83 \pm 0.06 \pm 0.08$ & & $0.71 \pm 0.05 \pm 0.07$ \\
\hline
\end{tabular}

shown in Fig. 3a. By unfolding the data with different generators and by using a bin-by-bin correction a mean spread per bin of $5.3 \%$ is observed and it is assigned to the systematic uncertainty. The total systematic uncertainty is estimated to be $9.6 \%$, almost independent of $x$.

The resulting values of $F_{2}^{\gamma}(x) / \alpha$ at $Q^{2}=120$ $\mathrm{GeV}^{2}$ can be compared with various calculations. In Fig. 7a a comparison is shown with the QPM predictions [3], with the QCD models GRV [25] and AGF [26] and with the LRSN next-to-leading order (NLO) QCD calculations [27]. As already observed by comparing the $x_{\text {fit }}$ distribution of data with Monte Carlo, at high $x$ the data are well described by the QPM, but QCD contributions are necessary for $x<0.5$. The existing QCD models predict similar values, but all are below the experimental data at low $x$.

The value of $F_{2}^{\gamma}(x) / \alpha$ at $\langle x\rangle=0.13$ can be compared to our measurements at lower values of $Q^{2}$ [7]. The $\ln Q^{2}$ evolution obtained there extrapolates to a value $F_{2}^{\gamma} / \alpha=0.63 \pm 0.13$ (stat.) \pm
0.13 (sys.) at $Q^{2}=120 \mathrm{GeV}^{2}$, in agreement with the measured value. Fig. $7 \mathrm{~b}$ and Table 4 show the $Q^{2}$-dependence of $F_{2}^{\gamma} / \alpha$ for the data, the QPM model [3] and the LRSN QCD calculation after integration over the range $x=0.05-0.98$. The data are higher than the model predictions. The QPM prediction depends on the assumed values of the quark masses. If the quark masses are reduced by $20 \%$, the average value of $F_{2}^{\gamma} / \alpha$ increases by about $5 \%$. The $Q^{2}$ evolution is compatible with a $\ln Q^{2}$ rise. For comparison with current other data [4-8], the results in the ranges $x=0.3-0.8$ and $x=0.1-0.6$ are given in Table 4 . We also give there $\left\langle F_{2}^{\gamma} / \alpha\right\rangle$ at $Q^{2}=120 \mathrm{GeV}^{2}$, averaged over the $Q^{2}$ range of the experiment.

\subsection{Double tag}

The procedure to derive the effective structure function $F_{\text {eff }}^{\gamma}(x)$ at average values $Q^{2}=120 \mathrm{GeV}^{2}$ and $P^{2}=3.7 \mathrm{GeV}^{2}$ is the same as that for single-tag

Table 5

$F_{\text {eff }}^{\gamma} / \alpha$ as function of $x$ for virtual photons at $P^{2}=3.7 \mathrm{GeV}^{2}, Q^{2}=120 \mathrm{GeV}^{2}$, from double-tag events.

\begin{tabular}{|c|c|c|c|c|c|}
\hline $\begin{array}{l}x \text { range } \\
\langle x\rangle\end{array}$ & $\begin{array}{r}0.05-0.2 \\
0.13\end{array}$ & $\begin{array}{r}0.2-0.4 \\
0.3\end{array}$ & $\begin{array}{r}0.4-0.6 \\
0.5\end{array}$ & $\begin{array}{r}0.6-0.8 \\
0.7\end{array}$ & $\begin{array}{r}0.8-0.98 \\
0.89\end{array}$ \\
\hline Ratio to QPM & $1.85 \pm 0.70$ & $1.63 \pm 0.56$ & $1.19 \pm 0.57$ & $1.89 \pm 0.76$ & $3.01 \pm 1.33$ \\
\hline $\begin{array}{l}F_{\text {eff }}^{\gamma}(x) / \alpha \\
\text { Systematic uncertainty }\end{array}$ & $\begin{array}{r}0.42 \pm 0.16 \\
0.05\end{array}$ & $\begin{array}{r}0.71 \pm 0.24 \\
0.09\end{array}$ & $\begin{array}{r}0.72 \pm 0.34 \\
0.09\end{array}$ & $\begin{array}{r}1.27 \pm 0.51 \\
0.16\end{array}$ & $\begin{array}{r}1.48 \pm 0.66 \\
0.19\end{array}$ \\
\hline $\begin{array}{l}\text { Correlation } \\
\text { matrix }\end{array}$ & 1 & $\begin{array}{c}-0.02 \\
1\end{array}$ & $\begin{array}{c}-0.08 \\
0.09 \\
1\end{array}$ & $\begin{array}{c}0.01 \\
-0.12 \\
0.12 \\
1\end{array}$ & $\begin{array}{r}0.01 \\
-0.04 \\
0.13 \\
0.50 \\
1\end{array}$ \\
\hline
\end{tabular}


data. The resulting values of $F_{\text {eff }}^{\gamma}(x) / \alpha$ are given in Table 5. The total systematic uncertainty is of the same order as that of the single-tag case; the selection is simpler and backgrounds are negligible, but the Monte Carlo and unfolding errors are larger. The total error is dominated by the statistical error.

In Fig. 8a the data on $F_{\text {eff }}^{\gamma} / \alpha$ are compared with the QPM and the GRS QCD model [28]. The data are higher than the QPM prediction, but still compatible with it within the combined statistical and systematic uncertainties. The sensitivity of the QPM prediction to the c-quark mass, and at values of $x$ larger than 0.9 to the u-quark mass, is similar to that for the single-tag case. At lower values of $x$ the sensitivity to the value of the u-quark mass is negligible. A reduction of QCD effects has been predicted
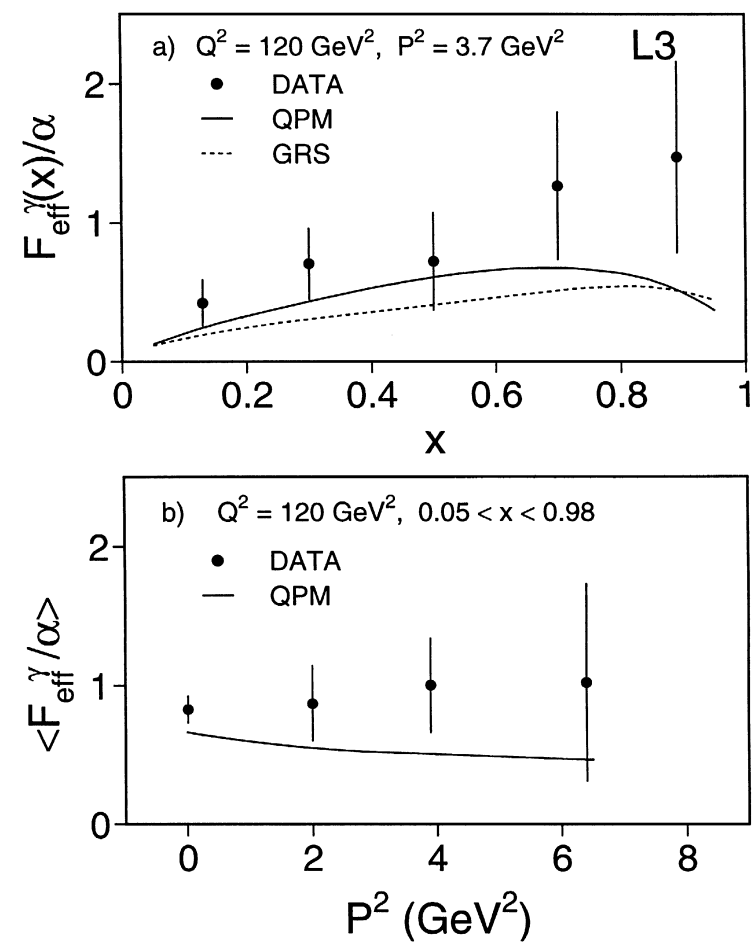

Fig. 8. (a) $F_{\text {eff }}^{\gamma} / \alpha$ for virtual photons at $Q^{2}=120 \mathrm{GeV}^{2}$ and $P^{2}=3.7 \mathrm{GeV}^{2}$, compared with the QPM calculation and the QCD calculation described in the text. The QCD calculation considers only transverse photons; therefore it is not really comparable with the double-tag data. (b) Dependence on $P^{2}$ of $F_{\text {eff }}^{\gamma} / \alpha$ averaged over $x=0.05-0.98$ for single-tag and double-tag data at $Q^{2}=120 \mathrm{GeV}^{2}$, compared with the QPM prediction, indicated as a full line. The errors are statistical and systematic added in quadrature.
Table 6

$P^{2}$ dependence of $F_{\text {eff }}^{\gamma} / \alpha$ for virtual photons at $Q^{2}=120 \mathrm{GeV}^{2}$. In the last row the structure function value is given for the total sample of double-tag events.

\begin{tabular}{ll}
\hline$\left\langle P^{2}\right\rangle$ & $\left\langle F_{\text {eff }}^{\gamma} / \alpha\right\rangle$ \\
$\left(\mathrm{GeV}^{2}\right)$ & $x=0.05-0.98$ \\
\hline 0 & $0.83 \pm 0.06 \pm 0.08$ \\
2.0 & $0.87 \pm 0.25 \pm 0.11$ \\
3.9 & $1.00 \pm 0.32 \pm 0.13$ \\
6.4 & $1.02 \pm 0.70 \pm 0.13$ \\
3.7 & $0.94 \pm 0.19 \pm 0.12$ \\
\hline
\end{tabular}

by Uematsu and Walsh [29], as the virtuality of the target photon limits gluon emission. In the present data their condition $Q^{2} \gg P^{2} \gg \Lambda^{2} \overline{\mathrm{MS}}$ is fulfilled, as $Q^{2}=120 \mathrm{GeV}^{2}, \quad P^{2}=3.7 \mathrm{GeV}^{2}$ and $\Lambda^{2} \overline{\mathrm{MS}} \approx 0.04$ $\mathrm{GeV}^{2}$. The GRS calculation pertains to the structure functions of transverse photons only, neglecting completely the longitudinal photon cross section; it is therefore impossible to draw a conclusion on the QCD behaviour by comparing this calculation to the data.

Fig. $8 \mathrm{~b}$ and Table 6 show the $P^{2}$-dependence of $F_{\text {eff }}^{\gamma} / \alpha$ averaged over the available $x$-range. The average value at $P^{2}=3.7 \mathrm{GeV}^{2}$ is also listed in the Table . The data are above QPM expectations, but compatible within the combined statistical and systematic uncertainties.

\section{Conclusions}

Two photon events $\mathrm{e}^{+} \mathrm{e}^{-} \rightarrow \mathrm{e}^{+} \mathrm{e}^{-}$hadrons have been studied in the high $Q^{2}$ range $40 \mathrm{GeV}^{2} \leq Q^{2} \leq$ $500 \mathrm{GeV}^{2}$ at $\sqrt{s} \simeq 91 \mathrm{GeV}$ in single-tag $\left(P^{2} \approx\right.$ $\left.0 \mathrm{GeV}^{2}\right)$ and double-tag $\left(1 \mathrm{GeV}^{2} \leq P^{2} \leq 8 \mathrm{GeV}^{2}\right)$ mode.

The event shape distributions are well described by underlying point-like interactions $\gamma \gamma \rightarrow \mathrm{q} \overline{\mathrm{q}}$ (QPM), $\gamma \mathrm{g} \rightarrow \mathrm{q} \overline{\mathrm{q}}, \gamma \mathrm{q} \rightarrow \mathrm{gq}$ (QCD), whereas there is no evidence of a strong contribution of VDM and diffractive components. The QPM diagram alone is insufficient to describe the observed $x$ distribution; a QCD contribution at low values of $x$ improves the agreement with the data.

The structure function $F_{2}^{\gamma}(x)$ of real photons shows an excess at low- $x$ over QPM and over sev- 
eral QCD calculations. The observed $Q^{2}$ evolution is compatible with the $\ln Q^{2}$ dependence measured previously [7] at lower $Q^{2}$ values.

An effective structure function is measured with double-tag events. The value is higher than predicted by the QPM, but still compatible within the combined statistical and systematic uncertainties.

\section{Acknowledgements}

We express our gratitude to the CERN accelerator divisions for the excellent performance of the LEP machine. We acknowledge with appreciation the effort of the engineers, technicians and support staff who have participated in the construction and maintenance of this experiment. We wish to thank E. Laenen, A. Vogt and M. Stratmann for useful discussions.

\section{References}

[1] E. Witten, Nucl. Phys. B 120 (1977) 189; C.H. Llewellyn Smith, Phys. Lett. B 79 (1978) 83; C.T. Hill, G.G. Ross, Nucl. Phys. B 148 (1979) 373; R. de Witt et al., Phys. Rev. D 19 (1979) 2046; W.R. Frazer, J.F. Gunion, Phys. Rev. D 20 (1979) 147.

[2] A. Vogt, The parton structure of real photons, Proc. of Photon'97, World Scientific, Singapore, 1997, p. 3 and references therein.

[3] V.M. Budnev, I.F. Ginzburg, G.V. Meledin, V.G. Serbo, Phys. Rep. C 15 (1975) 181.

[4] PLUTO Collaboration, Ch. Berger et al., Phys. Lett. B 107 (1981) 168; Nucl. Phys. B 281 (1987) 365. JADE Collaboration W. Bartel et al., Phys. Lett. B 121 (1983) 203; Z. Phys. C 24 (1984) 231. CELLO Collaboration, H.J. Behrend et al., Phys. Lett. B 126 (1983) 391. TASSO Collaboration, M. Althoff et al., Z. Phys. C 31 (1986) 527. TPC $/ 2 \gamma$ Collaboration, H. Aihara et al., Z. Phys. C 34 (1987) 1; Phys. Rev. Lett. 58 (1987) 97. AMY Collaboration, T. Sasaki et al., Phys. Lett. B 252 (1990) 491. TOPAZ Collaboration, K. Muramatsu et al., Phys. Lett. B 332 (1994) 477.
[5] OPAL Collaboration, R. Akers et al., Z. Phys. C 61 (1994) 199; OPAL Collaboration, K. Ackerstaff et al., Phys. Lett. B 411 (1997) 387; Phys. Lett. B 412 (1997) 225; Z. Phys. C 74 (1997) 33.

[6] DELPHI Collaboration, P. Abreu et al., Z. Phys. C 69 (1996) 223.

[7] L3 Collaboration, M. Acciarri et al., Phys. Lett. B 436 (1998) 403; Phys. Lett. B 447 (1999) 147.

[8] ALEPH Collaboration, R. Barate et al., Phys. Lett. B 458 (1999) 152.

[9] PLUTO Collaboration, Ch. Berger et al., Phys. Lett. B 142 (1984) 119.

[10] F.A. Berends et al., Comp. Phys. Comm. 40 (1986) 285.

[11] L3 Collaboration, B. Adeva et al., Nucl. Instr. Meth. A 289 (1990) 35; Nucl. Instr. Meth. A 351 (1994) 300.

[12] P. Béné et al., Nucl. Instr. Meth. A 306 (1991) 150.

[13] R. Bizzarri et al., Nucl. Instr. Meth. A 283 (1989) 799.

[14] JAMVG, J.A.M. Vermaseren, Nucl. Phys. B 229 (1983) 347.

[15] PHOJET version $1.05 \mathrm{c}$ is used. R. Engel, Z. Phys. C 66 (1995) 203; R. Engel, J. Ranft, Phys. Rev. D 54 (1996) 4244.

[16] J.H. Field, F. Kapusta, L. Poggioli, Phys. Lett. B 181 (1986) 362; Z. Phys. C 36 (1987) 121.

[17] TWOGAM version 1.71 is used. S. Nova, T. Todorov, DELPHI Note 90-35 (1990). We thank our colleagues from DELPHI for making the program available to us.

[18] T. Sjöstrand, Comp. Phys. Comm. 82 (1994) 74.

[19] S. Jadach, Comp. Phys. Comm. 79 (1994) 503.

[20] E. Farhi, Phys. Rev. Lett. 39 (1977) 1587.

[21] S.J. Brodsky et al., Phys. Rev. D 19 (1979) 1418; K. Kajantie, R. Raitio, Nucl. Phys. B 159 (1979) 528.

[22] A. Hoecker, V. Kartvelishvili, Nucl. Instr. Meth. A 372 (1996) 469. We thank V. Kartvelishvili for making his Fortran program GURU available to us.

[23] F.A. Berends et al., Comp. Phys. Comm. 40 (1986) 271; F.A. Berends et al., Nucl. Phys. B 253 (1985) 421.

[24] E. Laenen, G.A. Schuler, CERN-TH-97-193, Aug. 1997, hep-ph/9708261.

[25] M. Glück, E. Reya, A. Vogt, Phys. Rev. D 46 (1992) 1973.

[26] P. Aurenche, J.-P. Guillet, M. Fontannaz, Z. Phys. C 64 (1994) 621

[27] E. Laenen, S. Riemersma, J. Smith, W.L. van Neerven, Phys. Rev. D 49 (1994) 5753; E. Laenen, S. Riemersma, Phys. Lett. B 376 (1996) 169.

[28] M. Glück, E. Reya, M. Stratmann, Phys. Rev. D 54 (1996) 5515.

[29] T. Uematsu, T.F. Walsh, Phys. Lett. B 101 (1981) 263; Nucl. Phys. B 199 (1982) 93. 